\title{
Epstein-Barr virus-specific adoptive immunotherapy: a new horizon for multiple sclerosis treatment?
}

\begin{abstract}
"The beneficial effect of Epstein-Barr virus (EBV)-specific adoptive immunotherapy in this first patient with progressive multiple sclerosis [16] provides supportive evidence for a pathogenic role of EBV, and of decreased CD8 ${ }^{+} \mathrm{T}$-cell immunity to $E B V$, in the development of multiple sclerosis."
\end{abstract}

\begin{abstract}
Keywords: adoptive immunotherapy $\bullet$ B cell $\bullet$ CD8 T cell $\bullet$ Epstein-Barr virus - multiple sclerosis • treatment
\end{abstract}

Multiple sclerosis (MS) is a common chronic inflammatory demyelinating disease of the CNS causing progressive disability and affecting 2.5 million people worldwide. Usually, the disease has a relapsing-remitting course, with repeated neurologic episodes, each of which is followed by partial or complete recovery and a period free of new symptoms. Most patients with relapsingremitting MS eventually develop secondary progressive MS, in which there is progressive deterioration independent of relapses. In approximately $10 \%$ of patients, MS follows a primary progressive course, with a progressive neurologic deterioration from the onset, sometimes with superimposed relapses. Currently, there is no effective disease-modifying therapy for progressive MS.

Over the last 30 years, there has been increasing evidence that Epstein-Barr virus (EBV) has a role in the pathogenesis of MS $[1,2]$. EBV infection appears to be present in $100 \%$ of MS patients when two independent methods are used to determine EBV seropositivity [3]. Prospective studies have shown that primary EBV infection occurs on average 5.6 years before the onset of MS [4] and that high titers of serum IgG antibodies to EBV nuclear antigen-1 (EBNA1) increase the risk of developing MS [5,6]. Infectious mononucleosis also increases the risk of MS [7].

In 2003, the novel hypothesis was proposed that human chronic autoimmune diseases, including MS, are caused by EBV infection of autoreactive $B$ cells, which accumulate in the target organ where they produce pathogenic autoantibodies and provide costimulatory survival signals to autoreactive $T$ cells that would otherwise die in the target organ by activation-induced apoptosis [8]. It also postulates that the accumulation of EBV-infected autoreactive $\mathrm{B}$ cells in the target organ is due to a genetically determined defect in the elimination of EBV-infected B cells by the cytotoxic $\mathrm{CD}^{+}{ }^{+} \mathrm{T}$ cells that normally keep EBV infection under tight control. The hypothesis makes predictions that have subsequently been verified, namely: the presence of EBVinfected B cells in the brain in MS [9,10]; a beneficial effect in MS of rituximab, which kills $\mathrm{B}$ cells, including EBV-infected B cells [11]; decreased $\mathrm{CD}^{+}{ }^{+} \mathrm{T}$-cell immunity to EBV in MS [12]; and EBV infection of autoreactive plasma cells in the synovium in rheumatoid arthritis [13]. It also predicts that boosting CD8 ${ }^{+}$T-cell control of EBV by vaccination or by adoptive immunotherapy will prevent and successfully treat chronic autoimmune diseases.

AdE1-LMPpoly is a novel recombinant adenovirus vector encoding multiple $\mathrm{CD}^{+}$ T-cell epitopes from three EBV latent proteins, namely EBNA1, latent membrane protein (LMP) 1 and LMP2A [14]. Adoptive immunotherapy with autologous $\mathrm{T}$ cells expanded in vitro with AdE1-LMPpoly increases survival in patients with metastatic nasopharyngeal carcinoma, a disease in which the carcinoma cells are infected with EBV and express EBNA1, LMP1 and LMP2A [14]. Because EBV-infected $B$ cells in the brain in
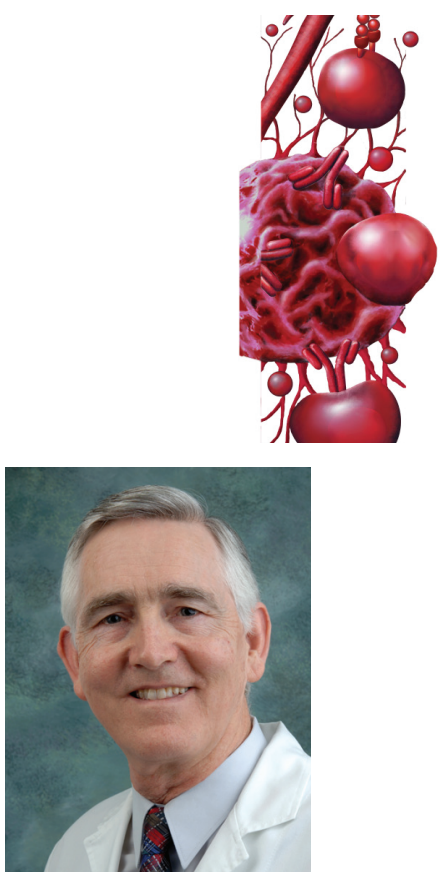

Michael P Pender

Author for correspondence: The University of Queensland, School of Medicine, Brisbane, Queensland, Australia

and

Department of Neurology, Royal Brisbane \& Women's Hospital, Brisbane, Queensland, Australia and

QIMR Centre for Immunotherapy \& Vaccine Development \& Department of Immunology, QIMR Berghofer Medical Research Institute, Brisbane, Queensland, Australia m.pender@uq.edu.au

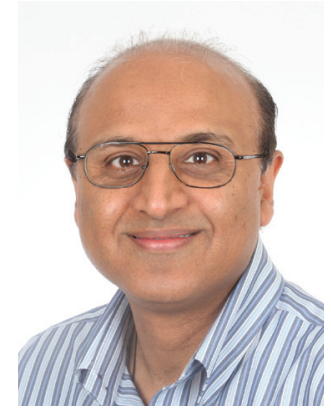

Rajiv Khanna

The University of Queensland, School of Medicine, Brisbane, Queensland, Australia

and

QIMR Centre for Immunotherapy \& Vaccine Development \& Department of Immunology, QIMR Berghofer Medical Research Institute, Brisbane, Queensland, Australia

Future $\because \%$ Medicine part of 
MS express the same three EBV proteins [9,15], adoptive immunotherapy with AdE1-LMPpoly may be an effective way to increase the number of $\mathrm{CD} 8^{+} \mathrm{T}$ cells available to eliminate EBV-infected B cells from the CNS in MS. Recently, we reported the first use of adoptive immunotherapy with AdE1-LMPpoly to treat a patient with MS [16].

The patient was a 42-year-old man with secondary progressive MS. His first attack of MS occurred in 1994 when he was IgG seropositive for EBNA and EBV viral capsid antigen but IgM seronegative for viral capsid antigen, indicating past infection with EBV. The course of his MS was relapsing-remitting until 2004, when it became secondary progressive. From 2000 to 2008, he was treated with IFN- $\beta-1$ b. Since 2008 , he had been unable to walk or transfer himself. By 2012, intention tremor was progressively limiting the use of his hands and he had a flexion contracture of the right knee. The proportion of EBV-specific CD8 ${ }^{+} \mathrm{T}$ cells in his blood was below the tenth percentile in healthy EBV carriers and he carried HLA-A2 and HLA-B7, which are restricting elements for several of the EBNA1, LMP1 and LMP2A epitopes in AdE1-LMPpoly. He also had the general $\mathrm{CD} 8^{+}$T-cell deficiency and an increased CD4:CD8 ratio typical of MS [17].

\section{"These beneficial effects of EBV-specific adoptive immunotherapy in our patient can be explained by the killing of EBV-infected B cells in the CNS by the adoptively transferred $\mathrm{CD} 8^{+} \mathrm{T}$ cells."}

This treatment was approved by the Royal Brisbane and Women's Hospital Clinical Ethical Review Group and through the Special Access Scheme (category B) of the Australian Government Therapeutic Goods Administration. With informed consent, we collected $400 \mathrm{ml}$ of blood and expanded his EBV-specific T cells by in vitro stimulation with AdE1-LMPpoly and IL-2 [14]. After expansion, $38.46 \%$ of CD8 ${ }^{+} \mathrm{T}$ cells but only $0.22 \%$ of $\mathrm{CD}^{+} \mathrm{T}$ cells reacted to the LMP peptides. The EBV-specific T cells were returned to the patient intravenously at fortnightly intervals. To reduce the risk of aggravating CNS inflammation, we chose an initial dose of $5 \times 10^{6} \mathrm{~T}$ cells, which was only $25 \%$ of the median dose used for nasopharyngeal carcinoma [14], and escalated the dose gradually over the following three infusions to $1 \times 10^{7}, 1.5 \times 10^{7}$ and $2 \times 10^{7}$ cells.

The treatment was successfully completed without significant adverse effects. In particular, there were no fevers, influenza-like symptoms or malaise. Following the treatment, he experienced a reduction in fatigue and painful lower limb spasms, an improvement in cognition and hand function, and increased productivity at work.
These improvements were sustained up to the time of the latest review, 21 weeks after the final T-cell infusion, when neurological examination demonstrated increased voluntary movement of his lower limbs. Following treatment the frequency of circulating EBV-specific $\mathrm{CD}^{+}$ $T$ cells increased and there were decreases in intrathecal IgG production and disease activity on magnetic resonance imaging of the brain.

These beneficial effects of EBV-specific adoptive immunotherapy in our patient can be explained by the killing of EBV-infected B cells in the CNS by the adoptively transferred $\mathrm{CD}^{+} \mathrm{T}$ cells. The EBVencoded LMP2A and LMP1 proteins targeted by the transferred $\mathrm{CD}^{+} \mathrm{T}$ cells are crucial in allowing EBVinfected $\mathrm{B}$ cells to multiply and mature into memory $\mathrm{B}$ cells and plasma cells capable of producing large amounts of antibody. LMP2A and LMP1 mimic the antigen-activated $\mathrm{B}$-cell receptor and the activated CD40 receptor, respectively $[18,19]$. While the EBVinfected autoreactive B cells in the brain may be driving the autoimmune attack on the brain by producing pathogenic autoantibodies and providing costimulatory survival signals to autoreactive T cells [8], the autoimmune process itself could promote the survival, proliferation and differentiation of the EBV-infected autoreactive $\mathrm{B}$ cells by releasing CNS antigens and giving $\mathrm{CD}^{+}{ }^{+} \mathrm{T}$-cell help, which would complement the $\mathrm{B}$-cell receptor and CD40 receptor signaling already provided by LMP2A and LMP1, respectively [20] that is 'double signaling'. This could lead to a vicious circle wherein EBV-infected autoreactive B cells promote autoimmunity, which in turn promotes EBV infection in the CNS. Such extensive double signaling through the B-cell receptor and CD40 pathways in the target organ of patients with chronic autoimmune diseases could be a relatively new experience for EBV in its 40 million years of coevolution with primates. Further research is needed to determine whether EBVinfected B cells and plasma cells in the MS brain are autoreactive, as has recently been shown for EBVinfected plasma cells in the synovium of patients with rheumatoid arthritis [13].

The adoptive transfer of EBV-specific CD8 ${ }^{+} \mathrm{T}$ cells in MS is not without risk. The transferred $\mathrm{T}$ cells could aggravate inflammation in the CNS and actually worsen MS, either through cross-reactivity between EBV and CNS antigens or through bystander damage [2]. A Phase I clinical trial is needed to determine the safety of EBV-specific adoptive immunotherapy in a larger number of patients with progressive MS. In view of the potential risk of aggravating CNS inflammation, this therapy should probably not be tried yet in patients with relapsing-remitting MS for which a number of disease-modifying therapies are already available. 
Another important question is how long any beneficial effect of EBV-specific adoptive immunotherapy in MS is likely to last. Because the therapy does not correct the generalized $\mathrm{CD} 8^{+} \mathrm{T}$-cell deficiency that could underlie the impaired $\mathrm{CD}^{+} \mathrm{T}$-cell immunity to EBV in MS $[2,17]$, it is likely that EBV-specific CD8 ${ }^{+}$T-cell immunity may eventually wane again after the initial increase from immunotherapy. If such a decrease is accompanied by worsening of MS, consideration should be given to administering a further course of EBV-specific adoptive immunotherapy.

The beneficial effect of EBV-specific adoptive immunotherapy in this first patient with progressive MS [16] provides supportive evidence for a pathogenic role of EBV, and of decreased $\mathrm{CD}^{+}{ }^{+}$T-cell immunity to EBV, in the development of MS. In addition, our study has implications for the treatment of other

\section{References}

1 Ascherio A, Munger KL. Epstein-Barr virus infection and multiple sclerosis: a review. J. Neuroimmune Pharmacol. 5(3), 271-277 (2010).

2 Pender MP. The essential role of Epstein-Barr virus in the pathogenesis of multiple sclerosis. Neuroscientist 17(4), 351-367 (2011).

3 Pakpoor J, Disanto G, Gerber JE et al. The risk of developing multiple sclerosis in individuals seronegative for Epstein-Barr virus: a meta-analysis. Mult. Scler. 19(2), 162-166 (2013).

4 Levin LI, Munger KL, O’Reilly EJ, Falk KI, Ascherio A. Primary infection with the Epstein-Barr virus and risk of multiple sclerosis. Ann. Neurol. 67(6), 824-830 (2010).

5 Sundström P, Juto P, Wadell G et al. An altered immune response to Epstein-Barr virus in multiple sclerosis: a prospective study. Neurology 62(12), 2277-2282 (2004).

6 Levin LI, Munger KL, Rubertone MV et al. Temporal relationship between elevation of Epstein-Barr virus antibody titers and initial onset of neurological symptoms in multiple sclerosis. JAMA 293(20), 2496-2500 (2005).

7 Thacker EL, Mirzaei F, Ascherio A. Infectious mononucleosis and risk for multiple sclerosis: a metaanalysis. Ann. Neurol. 59(3), 499-503 (2006).

8 Pender MP. Infection of autoreactive B lymphocytes with EBV, causing chronic autoimmune diseases. Trends Immunol. 24(11), 584-588 (2003).

9 Serafini B, Rosicarelli B, Franciotta D et al. Dysregulated Epstein-Barr virus infection in the multiple sclerosis brain. J. Exp. Med. 204(12), 2899-2912 (2007).

10 Tzartos JS, Khan G, Vossenkamper A et al. Association of innate immune activation with latent Epstein-Barr virus in active MS lesions. Neurology 78(1), 15-23 (2012).

11 Hauser SL, Waubant E, Arnold DL et al. B-cell depletion with rituximab in relapsing-remitting multiple sclerosis. N. Engl. J. Med. 358(7), 676-688 (2008). chronic autoimmune diseases where EBV also has a pathogenic role [8].

\section{Financial \& competing interests disclosure}

The authors's study on Epstein-Barr virus-specific immunotherapy in the patient with progressive MS was supported by Multiple Sclerosis Research Australia, the Trish Multiple Sclerosis Research Foundation and the QIMR Rio-Tinto Flagship Program on Immunotherapy. MP Pender has no financial or competing interests. R Khanna holds a patent on the EpsteinBarr virus epitopes included in the AdE1-LMPpoly construct. The authors have no other relevant affiliations or financial involvement with any organization or entity with a financial interest in or financial conflict with the subject matter or materials discussed in the manuscript apart from those disclosed.

No writing assistance was utilized in the production of this manuscript.

Pender MP, Csurhes PA, Lenarczyk A, Pfluger CMM, Burrows SR. Decreased T cell reactivity to Epstein-Barr virus infected lymphoblastoid cell lines in multiple sclerosis. J. Neurol. Neurosurg. Psychiatry 80(5), 498-505 (2009).

13 Croia C, Serafini B, Bombardieri M et al. Epstein-Barr virus persistence and infection of autoreactive plasma cells in synovial lymphoid structures in rheumatoid arthritis. Ann. Rheum. Dis. 72(9), 1559-1568 (2013).

14 Smith C, Tsang J, Beagley L et al. Effective treatment of metastatic forms of Epstein-Barr virus-associated nasopharyngeal carcinoma with a novel adenovirus-based adoptive immunotherapy. Cancer Res. 72(5), 1116-1125 (2012).

15 Serafini B, Severa M, Columba-Cabezas S et al. Epstein-Barr virus latent infection and BAFF expression in B cells in the multiple sclerosis brain: implications for viral persistence and intrathecal B-cell activation. J. Neuropathol. Exp. Neurol. 69(7), 677-693 (2010).

16 Pender MP, Csurhes PA, Smith C et al. Epstein-Barr virusspecific adoptive immunotherapy for progressive multiple sclerosis. Mult. Scler. doi:10.1177/1352458514521888 (2014) (Epub ahead of print).

17 Pender MP, Csurhes PA, Pfluger CMM, Burrows SR. Decreased $\mathrm{CD}^{+} \mathrm{T}$ cell response to Epstein-Barr virus infected $\mathrm{B}$ cells in multiple sclerosis is not due to decreased HLA class I expression on B cells or monocytes. BMC Neurol. 11, 95 (2011).

18 Mancao C, Hammerschmidt W. Epstein-Barr virus latent membrane protein $2 \mathrm{~A}$ is a $\mathrm{B}$-cell receptor mimic and essential for B-cell survival. Blood 110(10), 3715-3721 (2007).

19 Rastelli J, Hömig-Hölzel C, Seagal J et al. LMP1 signaling can replace CD40 signaling in B cells in vivo and has unique features of inducing class-switch recombination to IgG1. Blood 111(3), 1448-1455 (2008).

20 Pender MP. Does Epstein-Barr virus infection in the brain drive the development of multiple sclerosis? Brain 132(12), 3196-3198 (2009). 